\title{
From Becker-Döring to Lifshitz-Slyozov: deriving the non-local boundary condition of a non-linear transport equation
}

\author{
Romain Yvinec ${ }^{1, a}$, Julien Deschamps ${ }^{2}$, and Erwan Hingant ${ }^{3, b}$ \\ ${ }^{1}$ PRC INRA UMR85, CNRS, UMR7247, Université François Rabelais, IFCE, F-37380 Nouzilly, France \\ ${ }^{2}$ DIMA, Università degli Studi di Genova, Italy. \\ ${ }^{3}$ Departamento de Matemática, Universidad Federal de Campina Grande, PB, Brasil.
}

\begin{abstract}
.
We investigate the connection between two classical models of phase transition phenomena, the (discrete size, Markov chain or infinite set of ODE) Becker-Döring equations and the (continuous size, PDE) Lifshitz-Slyozov equation. Contrary to previous studies, we use a weak topology that includes the boundary of the state space, allowing us to rigorously derive a boundary value for the Lifshitz-Slyozov model. This boundary condition depends on a particular scaling and is the result of a separation of time scales.
\end{abstract}

\section{Introduction}

We address the link between two models of coagulation-fragmentation describing different stages of cluster growth. The Becker-Döring (BD) model [1] represents the microscopic stages, and describes the size repartition of clusters (or aggregates) using a discrete structure variable $i \in \mathbb{N}^{*}$, according to the set of chemical reactions

$$
C_{1}+C_{i} \underset{b_{i+1}}{\stackrel{a_{i}}{\rightleftharpoons}} C_{i+1}, \quad i \geq 1
$$

where $C_{i}$ stands for the clusters consisting of $i$ particles and $C_{1}$ the free particles. Here, the coefficients $a_{i}>0$ and $b_{i+1}>0$ denote respectively the rates of aggregation and fragmentation.

In the limit of a large number of particles, the time evolution of the concentration of clusters subject to the set of chemical reactions (1) is given by the solution of an infinite system of ordinary differential equations. Namely, the deterministic BD model is given, for all $t \geq 0$, by

$$
\left\{\begin{aligned}
\frac{d}{d t} C_{1}(t) & =-2 j_{1}(t)-\sum_{i \geq 2} j_{i}(t), \\
\frac{d}{d t} C_{i}(t) & =j_{i-1}(t)-j_{i}(t), \quad i \geq 2,
\end{aligned}\right.
$$

with

$$
j_{i}(t)=a_{i} C_{1}(t) C_{i}(t)-b_{i+1} C_{i+1}(t), \quad i \geq 1,
$$

\footnotetext{
a e-mail: correspondingauthor:romain.yvinec@tours.inra.fr

${ }^{b}$ Funded by CAPES/IMPA from Brazil.
} 
where the restriction $a_{i}=O(i)$ is imposed to ensure global existence, see [2,7]. The initial condition is subjected to $\left(C_{i}(0)\right)_{i \geq 1} \subset \mathbb{R}^{+}$, and $\sum_{i \geq 1} i C_{i}(0)=: M<\infty$.

Alternatively, considering a finite number of particles, such model (1) may be interpreted mathematically as a Markov Chain on a finite subset of a lattice. Denoting by $Y_{i}^{+}$(resp. $Y_{i}^{-}$) the standard Poisson process associated to the aggregation (resp. fragmentation) reaction of clusters of size $i \geq 1$ (resp. $i \geq 2$ ), the stochastic version of the BD model is given for $t \geq 0$ by

$$
\left\{\begin{array}{l}
C_{1}(t)=C_{1}(0)-2 J_{1}(t)-\sum_{i \geq 2} J_{i}(t), \\
C_{i}(t)=C_{i}(0)+J_{i-1}(t)-J_{k}(t), \quad i \geq 2,
\end{array}\right.
$$

with

$$
J_{i}(t)=Y_{i}^{+}\left(\int_{0}^{t} a_{i} C_{1}(s)\left(C_{i}(s)-\delta_{i}^{1}\right) d s\right)-Y_{i+1}^{-}\left(\int_{0}^{t} b_{i+1} C_{i+1}(s) d s\right), \quad i \geq 1,
$$

where $\delta_{i}^{1}=1$ if $i=1$ and $\delta_{i}^{1}=0$ if $i \geq 2$. The initial condition is subjected to $\left(C_{i}(0)\right)_{i \geq 1} \subset \mathbb{N}$, and $\sum_{i \geq 1} i C_{i}(0)=: M<\infty$.

The second model is the Lifshitz-Slyozov (LS) model $[3,6,8]$, and describes the cluster growth at a macroscopic scale. The size of the clusters are represented by a continuously varying variable $x>0$. If $f(t, x)$ denotes the size distribution function, the LS model is given by

$$
\begin{array}{ll}
\partial_{t} f(t, x)+\partial_{x}[(a(x) u(t)-b(x)) f(t, x)]=0, & t \geq 0, x>0, \\
u(t)+\int_{0}^{\infty} x f(t, x)=\text { const. }=: m, & t \geq 0,
\end{array}
$$

where $a(x)$ and $b(x)$ are respectively the aggregation and fragmentation rates. Note that in such a model, $u(t)$ plays the analog role of the concentration of free particles $C_{1}(t)$ in the BD model. If $a(x) u(t)-b(x)>0$ near $x=0$, such formulation lacks a proper boundary condition at $x=0$. It is the goal of this work to derive such boundary condition, using a rescaling of either Eq. (2) or Eq. (4).

\section{Rescaling and results}

\subsection{Rescaled equations}

The classical approach to operate a scaling is to write the equations in a dimensionless form. We follow [9] and introduce the following characteristic values:

$\bullet \bar{T}$ : characteristic time,

$-\bar{C}_{1}$ : characteristic value for $C_{1}$,

$\bullet \bar{C}$ : characteristic value for $C_{i}$, for $i \geq 2$,

$-\bar{M}_{c}$ : characteristic value for $M$,
- $\bar{A}_{1}$ : characteristic value for $a_{1}$,

- $\bar{B}_{2}$ : characteristic value for $b_{2}$,

$\bullet \bar{A}$ : characteristic value for $a_{i}, i \geq 2$,

$\bullet \bar{B}$ : characteristic value for $b_{i}, i \geq 3$.

We introduce the scaling parameter $\varepsilon>0$ and assume the following choices of relation:

$$
\bar{C} / \bar{C}_{1}=\varepsilon^{2}, \quad \bar{A} \bar{C}_{1} \bar{T}=\bar{B} \bar{T}=\frac{1}{\varepsilon}, \quad \bar{M}_{c} / \bar{C}_{1}=1, \quad \bar{A}_{1}=\varepsilon^{2} \bar{A}, \quad \bar{B}_{2}=\varepsilon^{\eta} \bar{B},
$$

with $\eta \in[0,1]$ to be chosen later. Thus, we define the dimensionless quantities

$$
\tau=t / \bar{T}, \quad u^{\varepsilon}(\tau)=C_{1}(\tau \bar{T}) / \bar{C}_{1}, \quad c_{i}^{\varepsilon}(\tau)=C_{i}(\tau \bar{T}) / \bar{C}, \quad m^{\varepsilon}=M / \bar{M}_{c},
$$


and (we use different letters at the boundary to emphasize this point)

$$
\alpha^{\varepsilon}:=a_{1} / \bar{A}_{1}, \quad \beta^{\varepsilon}:=b_{2} / \bar{B}_{2}, \text { and } a_{i}^{\varepsilon}=a_{i} / \bar{A}, \quad b_{i+1}^{\varepsilon}=b_{i+1} / \bar{B}, \quad i \geq 2 .
$$

We define the macroscopic reaction rates and fluxes

$$
a^{\varepsilon}(x):=\sum_{i \geq 2} a_{i}^{\varepsilon} \mathbf{1}_{\Lambda_{i}^{\varepsilon}}(x), \quad b^{\varepsilon}(x):=\sum_{i \geq 3} b_{i}^{\varepsilon} \mathbf{1}_{\Lambda_{i}^{\varepsilon}}(x), \text { and } j^{\varepsilon}(\tau, x)=\sum_{i \geq 2}\left(a_{i}^{\varepsilon} u^{\varepsilon}(\tau)-b_{i+1}^{\varepsilon}\right) \mathbf{1}_{\Lambda_{i}^{\varepsilon}}(x),
$$

where $\Lambda_{i}^{\varepsilon}=[(i-1 / 2) \varepsilon,(i+1 / 2) \varepsilon)$. The clusters quantities given by (2) (resp. by (4)) are now represented by a continuously varying variable $x>0$, and we let, for all $t \geq 0$ and all $\varepsilon>0$,

$$
f^{\varepsilon}(\tau, x)=\sum_{i \geq 2} c_{i}^{\varepsilon}(\tau) \mathbf{1}_{\Lambda_{i}^{\varepsilon}}(x), \quad x \geq 0
$$

Hence, using relations (7)-(8)-(9) the deterministic BD system (2)-(3) reads, for all $\tau \geq 0$,

$$
\left\{\begin{aligned}
\frac{d}{d \tau} u^{\varepsilon} & =-\varepsilon j_{1}^{\varepsilon}-\varepsilon \sum_{i \geq 1} j_{i}^{\varepsilon}, \\
\frac{d}{d \tau} c_{i}^{\varepsilon} & =\frac{1}{\varepsilon}\left[j_{i-1}^{\varepsilon}-j_{i}^{\varepsilon}\right], \quad i \geq 2,
\end{aligned}\right.
$$

where the rescaled fluxes are defined by

$$
j_{1}^{\varepsilon}=\alpha^{\varepsilon}\left(u^{\varepsilon}\right)^{2}-\beta^{\varepsilon} \varepsilon^{\eta} c_{2}^{\varepsilon}, \text { and } j_{i}^{\varepsilon}=a_{i}^{\varepsilon} u^{\varepsilon} c_{i}^{\varepsilon}-b_{i+1}^{\varepsilon} c_{i+1}^{\varepsilon}, \quad i \geq 2 .
$$

Then, for each $\varepsilon>0$ and all $\varphi \in W_{l o c}^{1, \infty}$ such that $\partial_{x} \varphi \in L^{\infty}$, we have from Eq. (11)-(12) for all $\tau \geq 0$

$$
\begin{aligned}
\int_{0}^{+\infty} f^{\varepsilon}(\tau, x) \varphi(x) d x= & \int_{0}^{+\infty} f^{i n, \varepsilon}(x) \varphi(x) d x+\int_{0}^{\tau} j_{1}^{\varepsilon}(\tau) \frac{1}{\varepsilon} \int_{\Lambda_{2}^{\varepsilon}} \varphi(x) d x d s \\
& +\int_{0}^{\tau} \int_{0}^{+\infty}\left[a^{\varepsilon}(x) u^{\varepsilon}(s) f^{\varepsilon}(s, x) \Delta_{\varepsilon} \varphi(x)-b^{\varepsilon}(x) f^{\varepsilon}(s, x) \Delta_{-\varepsilon} \varphi(x)\right] d x d s,
\end{aligned}
$$

with $\Delta_{h} \varphi(x)=(\varphi(x+h)-\varphi(x)) / h$ for $h \in \mathbb{R}$ and $u^{\varepsilon}(\tau)+\int_{0}^{\infty} x f^{\varepsilon}(\tau, x) d x=m^{\varepsilon}$.

Similarly, using additionally $\bar{C}=1$, with relations (7)-(8)-(9) the stochastic BD system (4)-(5) reads

$$
\left\{\begin{array}{l}
u^{\varepsilon}(\tau)=u^{\varepsilon}(0)-2 \varepsilon^{2} J_{1}^{\varepsilon}(\tau)+\sum_{i \geq 2} \varepsilon^{2} J_{i}^{\varepsilon}(\tau), \\
c_{i}^{\varepsilon}(\tau)=c_{i}^{\varepsilon}(0)+J_{i-1}^{\varepsilon}(\tau)-J_{i}^{\varepsilon}(\tau), \quad i \geq 2
\end{array}\right.
$$

with the rescaled flux

$$
\begin{aligned}
& J_{1}^{\varepsilon}(\tau)=Y_{1}^{+}\left(\int_{0}^{\tau} \varepsilon^{-1} \alpha^{\varepsilon} u^{\varepsilon}(s)\left(u^{\varepsilon}(s)-\varepsilon^{2}\right) d s\right)-Y_{2}^{-}\left(\int_{0}^{t} \varepsilon^{-1} \beta^{\varepsilon} \varepsilon^{\eta} c_{2}^{\varepsilon}(s) d s\right) \\
& J_{i}^{\varepsilon}(\tau)=Y_{i}^{+}\left(\int_{0}^{\tau} \varepsilon^{-1} a_{i}^{\varepsilon} u^{\varepsilon}(s) c_{i}^{\varepsilon}(s) d s\right)-Y_{i+1}^{-}\left(\int_{0}^{t} \varepsilon^{-1} b_{i+1}^{\varepsilon} c_{i+1}^{\varepsilon}(s) d s\right), \quad i \geq 2 .
\end{aligned}
$$

Then, from Eq. (14)-(15), we obtain the stochastic infinitesimal generator of $f^{\varepsilon}$ constructed by Eq. (10),

$$
\begin{aligned}
\mathcal{L}^{\varepsilon} \psi(f)=\frac{\psi\left(f+\mathbf{1}_{\Lambda_{2}^{\varepsilon}}\right)-\psi(f)}{\varepsilon} \alpha^{\varepsilon} u^{\varepsilon}\left(u^{\varepsilon}-\varepsilon^{2}\right)+\frac{\psi\left(f-\mathbf{1}_{\Lambda_{i}^{\varepsilon}}\right)-\psi(f)}{\varepsilon} \beta^{\varepsilon} \varepsilon^{\eta} c_{2}^{\varepsilon} \\
+\sum_{i \geq 2} \frac{\psi\left(f+\mathbf{1}_{\Lambda_{i+1}^{\varepsilon}}-\mathbf{1}_{\Lambda_{i}^{\varepsilon}}\right)-\psi(f)}{\varepsilon} a_{i}^{\varepsilon} u^{\varepsilon} c_{i}^{\varepsilon}+\sum_{i \geq 3} \frac{\psi\left(f-\mathbf{1}_{\Lambda_{i}^{\varepsilon}}+\mathbf{1}_{\Lambda_{i-1}^{\varepsilon}}\right)-\psi(f)}{\varepsilon} b_{i}^{\varepsilon} c_{i}^{\varepsilon},
\end{aligned}
$$


where $c_{i}^{\varepsilon}=\varepsilon^{-1} \int_{\mathbf{1}_{\Lambda_{i}^{\varepsilon}}} f(t, x) d x, i \geq 2$, and $u^{\varepsilon}=m^{\varepsilon}-\int x f(t, x)$. In particular, with $\psi(f)=\langle f, \varphi\rangle$ and $\varphi \in C_{b}$, we obtain an $L^{2}$-martingale given by

$$
\begin{aligned}
\mathcal{M}^{\varepsilon, \tau, \varphi}:=\int_{0}^{+\infty} f^{\varepsilon}(\tau, x) \varphi(x) d x-\int_{0}^{+\infty} f^{i n, \varepsilon}(x) \varphi(x) d x \\
-\int_{0}^{\tau}\left[\alpha^{\varepsilon} u^{\varepsilon}(s)\left(u^{\varepsilon}(s)-\varepsilon^{2}\right)-\beta^{\varepsilon} \varepsilon^{\eta} c_{2}^{\varepsilon}(s)\right] \frac{1}{\varepsilon} \int_{\Lambda_{2}^{\varepsilon}} \varphi(x) d x d s \\
\quad-\int_{0}^{\tau} \int_{0}^{+\infty}\left[a^{\varepsilon}(x) u^{\varepsilon}(s) f^{\varepsilon}(s, x) \Delta_{\varepsilon} \varphi(x)-b^{\varepsilon}(x) f^{\varepsilon}(s, x) \Delta_{-\varepsilon} \varphi(x)\right] d x d s .
\end{aligned}
$$

\subsection{Results}

To obtain the convergence of $\left\{f^{\varepsilon}\right\}$, we use the measure space $\mathcal{X}:=\left\{v \in \mathcal{M}_{f}\left(\mathbb{R}^{+}\right): \int_{0}^{\infty} x v(d x)<+\infty\right\}$, equipped with the weak topology, that is $\left\{f^{\varepsilon}(x) d x\right\}$ converges to $\mu$ in $\mathcal{X}$ if

$$
\int_{0}^{\infty}(1+x) \varphi(x) f^{\varepsilon}(x) d x \rightarrow \int_{0}^{\infty}(1+x) \varphi(x) \mu(d x), \quad \forall \varphi \in C_{b}([0,+\infty)) .
$$

We assume standard convergence properties of the reactions rates $\alpha^{\varepsilon}, \beta^{\varepsilon}, a^{\varepsilon}(\cdot)$ and $b^{\varepsilon}(\cdot)$ towards (resp.) $\alpha, \beta, a(\cdot)$ and $b(\cdot)$, together with convergence of the initial condition $u^{\varepsilon}$ and $f^{i n, \varepsilon}(\cdot)$ towards (resp.) $u^{\text {in }}$ and $\mu^{i n}(\cdot)$. In order to derive the boundary condition, we need to know precisely the behavior of the rate functions near 0 . For that, we suppose there exist $r_{a}, r_{b} \in[0,1]$, and $\bar{a}, \bar{b}>0$ such that

$$
\begin{array}{l|l}
0 \leq r_{a}<1, & r_{a} \leq r_{b}, \\
a(x) \sim_{0^{+}} \bar{a} x^{r_{a}}, & b(x) \sim_{0^{+}} \bar{b} x^{r_{b}}, \\
a^{\varepsilon}(\varepsilon i)=a(\varepsilon i)+o\left((\varepsilon i)^{r_{a}}\right), i \geq 2, & b^{\varepsilon}(\varepsilon i)=b(\varepsilon i)+o\left((\varepsilon i)^{r_{b}}\right), i \geq 3 .
\end{array}
$$

where $o$ is the Landau notation in $\varepsilon \rightarrow 0^{+}$(independent on $i$ ). We also define the critical quantity

$$
\rho:=\lim _{x \rightarrow 0^{+}} \frac{b(x)}{a(x)}=\lim _{x \rightarrow 0^{+}} \frac{\bar{b}}{\bar{a}} x^{r_{b}-r_{a}} \in[0,+\infty) .
$$

Under reasonable assumptions on the macroscopic reaction rates, we are able to prove (see [4]) compactness property on $f^{\varepsilon}$ in $\mathcal{X}$ (for both the deterministic Eq. (13) and the stochastic Eq. (16)) Then, for any convergent subsequence, we prove that the candidate limit $\mu \in C([0, T] ; \mathcal{X})$ and $u \in C([0, T])$ satisfy, for all $\left.\varphi \in C_{c}^{1}(0,+\infty)\right)$ and $t \geq 0$

$$
\begin{aligned}
& \int_{0}^{\infty} \varphi(x) \mu(t, d x)=\int_{0}^{\infty} \varphi(x) \mu^{i n}(d x)+\int_{0}^{t} \int_{0}^{\infty} \varphi^{\prime}(x)(a(x) u(s)-b(x)) \mu(t, d x) d s, \\
& u(t)+\int_{0}^{\infty} x \mu(t, d x)=m .
\end{aligned}
$$

Note that Eq. (17) is formally the weak form of Eq. (6). Moreover, for any $T>0$ such that the limit satisfies $\inf _{t \in[0, T]} u(t)>\rho$, then for all $\varphi \in C_{b}^{1}([0,+\infty))$ and $t \geq 0$

$$
\langle\mu(t), \varphi\rangle=\left\langle\mu^{\mathrm{in}}, \varphi\right\rangle+\int_{0}^{t} \int_{0}^{\infty} \varphi^{\prime}(x)(a(x) u(s)-b(x)) \mu(t, d x) d s+\varphi(0) \int_{0}^{t} N(s) d s .
$$



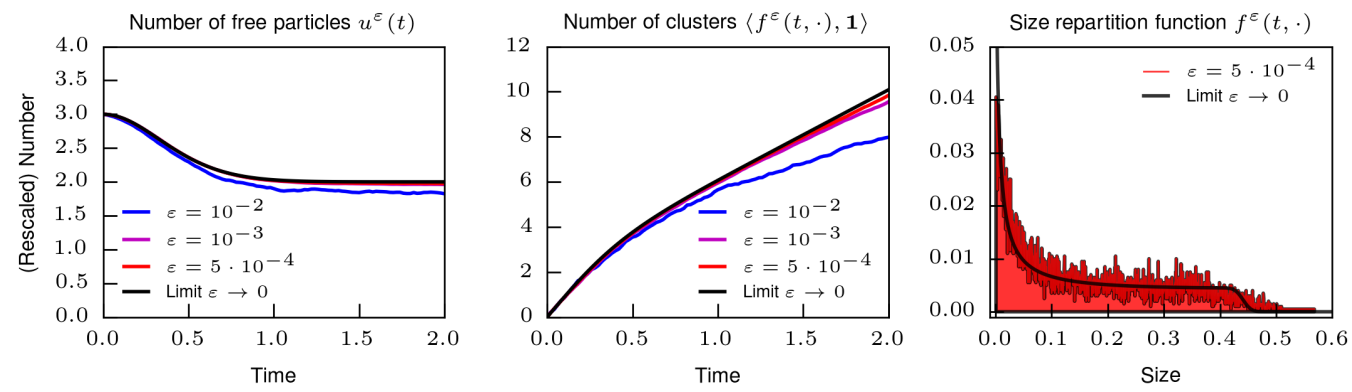

Figure 1. Agreement between numerical simulations and our limit theorem. We plot the time evolution of the (rescaled) number of free particles $u^{\varepsilon}(t)$ (left) and the total (rescaled) number of clusters $\left\langle f^{\varepsilon}(t, \cdot), \mathbf{1}\right\rangle$ (middle) for different $\varepsilon$ (see legend), together with the deterministic solution (in black) of the moment equations obtained from the weak form of the LS equation (18) with respectively $\varphi=x$ and $\varphi=1$. Right, we plot one snaphshot at time $t=1$ of the size distribution function $f^{\varepsilon}(t, \cdot)$ for $\varepsilon=5.10^{-4}$, together with the numerical solution of the LS equation (standard upwind scheme). We used the scaling given in Section 2.1 with constant rate coefficients $a^{\varepsilon}(x) \equiv 1$ and $b^{\varepsilon}(x) \equiv 2, \alpha^{\varepsilon}=1$, and $\beta^{\varepsilon}=1$, under incoming characteristics, i.e $u(0)=m=3>\rho=\frac{b}{a}=2$.

with for all $t \in[0, T]$

$$
N(t)= \begin{cases}\alpha u(s)^{2} & \text { if } \eta>r_{a} \\ \alpha u(s)^{2} \frac{2^{\eta} u(s)}{2^{\eta} u(s)+\beta} & \text { if } \eta=r_{a}<r_{b} \\ \alpha u(s)^{2} \frac{\bar{a} u(s)-\bar{b}}{\bar{a} u(s)-\bar{b}+\beta / 2^{\eta}} & \text { if } \eta=r_{a}=r_{b} \\ 0 & \text { if } \eta<r_{a}<r_{b}\end{cases}
$$

Thus the precise expression of the boundary condition Eq. (19) is dependent on the behavior of the rate functions near 0 and on the scaling factor $\eta$, that measures the relative speed of the fragmentation rate for $i=2$ with the fragmentation rates for $i>2$ (equal to $r_{b}$ for the latter). The case $\eta=1>r_{a}$ was treated for the stochastic Eq. (16) in [4] and all cases for the deterministic Eq. (13) in [5].

\section{Illustration and Discussion}

We illustrate our theoretical results with the help of a numerical simulation, see Figure 1. We show the good agreement between the rescaled solution of the stochastic BD system (14) and the limit given by Eq. (18). The importance of deriving such results is both numerical, in order to design fast numerical scheme to approximate large discrete system, and theoretical, to derive steady-state and time-dependent properties of the original discrete system from a (simpler) continuous one [10, 11]. In our scaling, each cluster of size initially $i \geq 2$ is seen as a cluster of size roughly $i \varepsilon \in \mathbb{R}_{+}$, and our scaling consists in an acceleration of the fluxes (by 1/ع) in Eq. (11) (resp. Eq. (14)) so that it can reach an "infinite" size $i=x / \varepsilon$ in finite time. This requires a large amount of free particles, of order $1 / \varepsilon^{2}$ compared to the amount of clusters. The first aggregation rate $a_{1}$ has been rescaled differently for the others for $i \geq 2$. This is due to the particular role played by the free particles in the BD model. Indeed, since we have assumed a large excess of free particles compared to the number of clusters (to 
form large ones), to keep such properties through time, one has to "slow down" the first nucleation rate $a_{1}$, that transform free particles into clusters (of size 2). If this is not satisfied, we hypothesize that the pool of free particles would deplenish, leading to a different version of the LS, namely the Lifshitz-Slyozov-Wagner model (work in progress, see also [7]).

From those previous work [7, 9], the originality of our work [4] resides in being able to derive the boundary condition of the limit equation. Such boundary condition is needed when the characteristics of the transport equation are incoming (i.e. the small clusters tend to grow). This result was obtained as an averaging result. In order to form large clusters, we have to accelerate the fluxes $($ by $1 / \varepsilon)$. Then, each individual size, and a fortiori the minimal size $i=2$, evolves in a fast time scale. By proving a quasi steady-state result, we were able to prove that each discrete size tend to equilibrate to a value given by the stationary state of an auxiliary system, very similar to the original deterministic BD model (but in a linear version).

Finally, second order approximation and large deviation phenomena of the stochastic discrete BD system (14) were observed numerically [10]. In particular, when the formation of large cluster is (asymptotically) very unlikely, the latter appears as a large deviation from the mean-field limit and gives a suitable framework to describe phase transition phenomena, as inherent infrequent stochastic processes, in contrast to classical nucleation theory. The precise quantification is left for future work.

\section{References}

[1] R. Becker and W. Döring, Kinetische behandlung der keimbildung in übersättigten dämpfen, Annalen der Physik 24 719-752 (1935).

[2] J.M. Ball,J. Carr and O. Penrose, The Becker-Döring Cluster Equations: Basic Properties and Asymptotic Behaviour of Solutions,

Commun. Math. Phys., 1044 (1986)

[3] J. Collet, T. Goudon and A. Vasseur, Some Remarks on Large-Time Asymptotic of the LifshitzSlyozov Equations, J. Stat. Phys., 108 1-2 (2002)

[4] J. Deschamps, E. Hingant and R. Yvinec, Boundary value for a nonlinear transport equation emerging from a stochastic coagulation-fragmentation type model, arXiv:1412.5025, (2015)

[5] J. Deschamps, E. Hingant and R. Yvinec, Quasi steady state approximation of the small clusters in Becker-Döring equations leads to boundary conditions in the Lifschitz-Slyozov limit, in preparation, (2015)

[6] P. Laurençot, The Lifshitz-Slyozov-Wagner Equation With Conserved Total Volume, SIAM J. Math. Anal., 342 (2002)

[7] P. Laurençot and S. Mischler, From the Becker-Döring to the Lifshitz-Slyozov-Wagner Equations, J. Stat. Phys., 106 5-6 (2002)

[8] B. Niethammer, Effective Theories for Ostwald Ripening, P. Mörters et al., Analysis and Stochastics of Growth Processes and Interface Models (Oxford University Press, 2008), 223-242.

[9] A. Vasseur, F. Poupaud, J-F Collet and T. Goudon, The Beker-Döring System and its LifshitzSlyozov Limit, SIAM J. Appl. Math., 625 (2002)

[10] R. Yvinec, S. Bernard, E. Hingant, L. Pujo-Menjouet, First passage times in homogeneous nucleation: dependence on the total number of particles arXiv:1510.04730, (2015)

[11] R. Yvinec, M. R. D'Orsogna and T. Chou, First passage times in homogeneous nucleation and self-assembly, J. Chem. Phys., 13724 (2012) 